\title{
Making pretest genomic counseling optional: lessons from the RAVE study
}

\author{
Erica J. Sutton, $\mathrm{PhD}^{1,2}$, Iftikhar J. Kullo, $\mathrm{MD}^{3,4}$ and Richard R. Sharp, $\mathrm{PhD}^{1,2}$
}

The successful integration of genomic medicine into clinical practice will require genetic professionals to reevaluate clinical standards that might have been appropriate when the numbers of individuals pursuing genomic sequencing were low, but may not be feasible on a larger scale. One such practice is the expectation that patients and research participants meet with a genetic counselor prior to pursuing genomic evaluation. Anticipating a time when genomic sequencing is an integral element of medical care, this expectation may be incompatible with counselor availability and patient interest in genetic counseling services. ${ }^{1,2}$

As part of the Return of Actionable Variants Empirical (RAVE) study, which is examining disclosure of results from targeted genomic sequencing of a panel of medically relevant genes, we are examining patients' interest in elective genetic counseling. The RAVE study will evaluate 109 genes, including the 59 genes identified as medically actionable by the American College of Medical Genetics and Genomics, in approximately 3,000 individuals. Actionable results will be disclosed to patients by a genetic professional and placed in their electronic health record.

In contrast to standard approaches to genomic sequencing, participants in the RAVE study were not required to receive pretest genetic counseling. Instead, genetic counseling was offered on an optional, no-cost basis to those individuals who expressed an interest. During the recruitment process, which occurred from 18 March 2016 to 29 April 2016, an invitation was mailed to all potential volunteers. This invitation included a 4-page informational pamphlet, a 2-page "frequently asked questions" document, and a 14-page consent form describing the study. These materials were developed by our genetic counseling team and included standard elements of a pretest genetic counseling session. These materials discussed how individuals were selected to participate in RAVE, why the study is being done, what participation requires, who has access to health information, what kinds of genetic test results will be generated, how participants will receive genetic test results, why someone would want to participate, what privacy protections are in place, potential implications for family members, and who to contact with questions about the study.
Each of these recruitment documents also mentioned the availability of elective genetic counseling services, which was framed in a neutral way that neither encouraged nor discouraged their use. For example, the informational pamphlet noted:

You may also speak with a genetic counselor if you have questions about the tests being performed in this study, implications of genetic test results for you and/or your family, or why you may or may not want to participate. To do so, call [telephone number] and ask to schedule a time to speak with a genetic counselor about the RAVE study.

A total of 4,788 adults were invited to participate in the RAVE study. Although our genetic counselors and study coordinators were prepared for hundreds of phone calls from potential volunteers, only 14 of the 4,788 invitees called with questions about the study (0.3\%) and just 8 of those individuals asked to speak with a genetic counselor (0.2\%). The 8 callers were primarily white, college-educated men between 47 and 69 years of age. A thematic analysis revealed that the most common motivation for speaking with a genetic counselor was to clarify whether participants could be denied health insurance based on sequencing results. Participants were also interested in learning more about the types of genetic results they might receive and the potential impact of those results on their health.

These findings highlight significant underutilization of optional pretest genetic counseling services. Individuals who went on to enroll in the RAVE study were later asked to complete a 13-page, 100-item survey to assess their decisionmaking process and expectations of the RAVE study. This survey consisted of both validated scales and de novo items created by the study team. Prior to finalizing the survey we conducted 14 cognitive interviews with participants in the Mayo Clinic Biobank.

Of the 2,898 RAVE participants who completed the survey ( $95.4 \%$ completion rate), 2,208 (76.5\%) reported that they made a decision to enroll immediately after reading the recruitment materials. Approximately one third of RAVE study participants $(36.2 \%, n=1,048)$ spoke with someone prior to making their decision to pursue genomic sequencing

${ }^{1}$ Biomedical Ethics Research Program, Mayo Clinic, Rochester, Minnesota, USA; ${ }^{2}$ Center for Individualized Medicine, Mayo Clinic, Rochester, Minnesota, USA; ${ }^{3}$ Department of Cardiovascular Diseases, Mayo Clinic, Rochester, Minnesota, USA; ${ }^{4}$ Gonda Vascular Center, Mayo Clinic, Rochester, Minnesota, USA. Correspondence: Richard R. Sharp (sharp. richard@mayo.edu)

Submitted 30 June 2017; accepted 20 November 2017; advance online publication 1 February 2018. doi:10.1038/gim.2017.240 
and just $1.4 \%(n=40)$ spoke with a health-care provider. Participants also completed a 16 -item decisional conflict scale. $^{3}$ Most participants self-reported that they understood the benefits $(83.9 \%, n=2,405)$ and risks $(81.2 \%, n=2,329)$ of pursuing genomic sequencing and felt they had received sufficient advice to make their decision $(71.7 \%, n=2,052)$. In addition, most participants self-reported that they felt prepared to cope with potential results they might receive, including results that identified an increased disease risk for which no preventative measures are available $(80.8 \%$, $n=2,341)$. Importantly, the vast majority of RAVE study participants $(95.8 \%, n=2,750)$ felt they had made an informed decision to pursue genomic sequencing.

These findings suggest that many people feel they can make an informed decision about whether to pursue genomic screening without the in-person support typically provided by a genetic counselor and that patient demand for elective genetic counseling may create less of a strain on genomic medicine than is believed. ${ }^{1,2,4}$ Although there will always be patients who need the individualized care and decisionmaking support that in-person genetic counseling sessions provide, ${ }^{5,6}$ many patients will not seek genetic counseling without stronger encouragement. ${ }^{7}$

Given the limited number of genetic counselors nationwide, and the increasing demands on their time, ${ }^{8}$ in-person genetic counseling sessions must be allocated wisely, with priority given to those individuals who are likely to have difficulty coping with test results or who are likely to regret their decision to pursue genomic testing. To identify those individuals who would benefit the most from such services, it is critical that future research explore the effectiveness of pretest screening tools that assess how confident patients are in their decision to pursue genomic sequencing and how prepared they are to receive various test results. One point of departure for the development of such a tool may be an abridged version of O'Connor's decisional conflict scale, a widely used measure of decisional ambivalence. ${ }^{3}$ This scale, or a similar screening tool, can promote informed decisionmaking in large-scale research and clinical genomic sequencing initiatives by redirecting individuals who might otherwise elect not to pursue optional genetic counseling.

As genomic medicine becomes more familiar to patients, and ultimately becomes a part of our shared health-care vocabulary, genetic counselors will need to re-envision their roles and consider nonconventional ways of supporting individuals pursuing genomic testing. Although the conventional approach to clinical genomic sequencing has been to encourage all patients to pursue in-person pretest genetic counseling, our findings highlight the importance of developing alternative strategies for educating and supporting large numbers of patients in clinical and research contexts. One such approach may be group counseling sessions, a strategy that has been used effectively in other contexts and might be adapted for genomic testing. ${ }^{9,10}$ Other approaches include the use of decision aids, Web-based educational content, and e-counseling tools, ${ }^{1}$ all of which might be especially useful mechanisms for patients making genomic sequencing decisions in clinical settings where comprehensive research consent forms and accompanying educational materials are not the norm. These pretest alternatives to in-person genetic counseling might be combined with an expectation that patients will meet with a genetic counselor following genomic sequencing to discuss test findings, thereby focusing scarce counseling resources on the interpretation and management of clinically actionable results. ${ }^{5}$ Future research is necessary to determine which of these alternatives to conventional pretest genetic counseling may be most effective.

\section{ACKNOWLEDGMENTS}

This project was supported by the National Human Genome Research Institute's Electronic Medical Records and Genomics (eMERGE) network (U01 HG006379) and Mayo Clinic's Center for Individualized Medicine. We thank Kylie Osterhus, Joel Pacyna, Jennifer McCormick, Erin Winkler, Emily Lisi, Carmen Radecki Breitkopf, and Marguerite Robinson for their contributions to this project.

\section{DISCLOSURE}

The authors declare no conflict of interest.

\section{REFERENCES}

1. Birch PH. Interactive e-counselling for genetics pre-test decisions: where are we now? Clin Genet 2015;87:209-217.

2. Darst BF, Madlensky L, Schork NJ, Topol EJ, Bloss CS. Perceptions of genetic counseling services in direct-to-consumer personal genomic testing. Clin Genet 2013;84:335-339.

3. O'Connor AM. Validation of a decisional conflict scale. Med Decis Making 1995;15:25-30.

4. Schmidlen TJ, Wawak L, Kasper R, Garcia-Espana JF, Christman MF, Gordon ES. Personalized genomic results: analysis of informational needs. J Genet Couns 2014;23:578-587.

5. Sie AS, Spruijt L, van Zelst-Stams WA, et al. High satisfaction and low distress in breast cancer patients one year after BRCA-mutation testing without prior face-to-face genetic counseling. J Genet Couns 2016;25: 504-514.

6. Suckiel SA, Linderman MD, Sanderson SC, et al. Impact of genomic counseling on informed decision-making among ostensibly healthy individuals seeking personal genome sequencing: the HealthSeq Project. J Genet Couns 2016;25:1044-1053.

7. Levin E, Riordan S, Klein J, Kieran S. Genetic counseling for personal genomic testing: optimizing client uptake of post-test telephonic counseling services. J Genet Couns 2012;21:462-468.

8. Sukenik-Halevy R, Ludman MD, Ben-Shachar S, Raas-Rothschild A. The time-consuming demands of the practice of medical genetics in the era of advanced genomic testing. Genet Med 2016;18:372-377.

9. Benusiglio PR, Di Maria M, Dorling L, et al. Hereditary breast and ovarian cancer: successful systematic implementation of a group approach to genetic counselling. Fam Cancer 2017;16:51-56.

10. Otten E, Birnie E, Ranchor AV, van Tintelen JP, van Langen IM. A group approach to genetic counselling of cardiomyopathy patients: satisfaction and psychological outcomes sufficient for further implementation. Eur J Hum Genet 2015;23:1462-1467. 